and the other in paediatrics. With an adequate backup of technical and non-medical staff to maintain the laboratory services two individuals should be able to run a specialised genetic service and retain an active clinical practice. While a scheme like this would not preclude from medical genetics the doctor who wished to give most of his time to the laboratory or research aspects, it would attract those recruits who wished to remain clinicians.

One of the most worrying trends in British medicine over the last few years has been the rigidity of the training programmes which have been set out by the royal colleges. These programmes are supposed to be flexible, but undoubtedly in many cases they are destroying initiative, and unusually bright young doctors who wish to pursue a career in a way which does not fit the pattern get out by the colleges are finding themselves in difficulties. In particular, the gifted young research worker finds it difficult to spend several years in clinical research and then get back on to the rather rigid career ladders. The training programme set out by the Clinical Genetics Society is excellent in many ways, but it should aim at even more flexibility at the specialist training stage. In particular, it should try to ensure that at least some specialists in medical genetics can maintain a strong clinical association with any medical specialty that they choose and that there is easy access to the discipline for those who have spent several years in full-time research.

A subject as diverse as medical genetics will lose much of its excitement and intellectual stimulation if the training programme becomes too rigid or the clinical content for consultants is too limited. The specialty should aim at a healthy mix of clinicians in many topics, laboratory workers, and nonmedical scientists. Haematology and immunology are two examples of fields that are suffering from rather rigid training programmes with limited opportunities for those with a strong clinical or research bent. The geneticists should be careful not to follow suit.

\section{Smoker's heart}

The harmful effects of cigarette smoking on the arteries are well known, the best recorded association being Buerger's disease (thromboangiitis obliterans)-a condition of unknown aetiology which is severely aggravated by smoking. Atheroma is also made worse by smoking ${ }^{1}$; the increased incidence and severity of coronary artery disease (as shown by myocardial infarction with an associated higher death rate) are well documented among heavy cigarette smokers. ${ }^{2}$ Recently Auerbach and his colleagues have investigated post mortem the changes in the coronary arteries and arterioles of heavy smokers and compared them with the changes encountered in milder smokers and in those who did not smoke at all. ${ }^{3}$ In this survey they excluded all patients who had suffered from diabetes mellitus and also those whose hearts weighed over 500 grams, since patients with hearts heavier than that would probably have severe hypertension, which itself accentuates atheroma.

Their results confirmed the increased incidence of severe atheroma in heavy smokers: in those who had used two or more packs a day there was four times as much atheroma as in the group that had never smoked regularly. Of even greater interest was the presence of fibrous intimal thickening of the wall of the coronary arteries. This microscopical change was found in advanced degree in nearly one-quarter of those who had smoked two or more packs a day as compared with less than $1 \%$ of those who had never smoked. Atheroma was found only in those cases that showed fibrous thickening, but fibrous thickening was frequently found in the absence of atheroma.

It was, however, the myocardial arterioles that showed the most severe effects of cigarette smoking. Advanced hyaline thickening was found in $90 \%$ of those who had smoked two or more packs a day, whereas in those who had smoked one to two packs a day the incidence was just less than half. The most interesting finding was the complete absence of hyaline change in the myocardial arterioles in any member of the group that never smoked regularly. Auerbach and his colleagues had previously shown an association between hyaline thickening of the arterioles and cigarette smoking in other organs also, ${ }^{4}$ and had produced it experimentally in dogs. ${ }^{5}$ They suggest that appreciable hyaline thickening in the myocardial arterioles with or without the associated fibrous intimal thickening in larger coronary arteries may properly be called the "smoker's heart."

How these morphological changes are produced is unknown. One possibility is the effect of nicotine, since the death rate from coronary arterial disease is less in people who smoke low $\operatorname{tar}$ /nicotine cigarettes. ${ }^{6}$ Dogs given these cigarettes to smoke show a decreased amount of hyaline thickening of their myocardial arterioles. ${ }^{5}$ Nicotine causes the release of adrenaline, and patients with phaeochromocytoma show changes in their myocardial arterioles. ${ }^{7}$ According to this theory the vasoconstrictive effect would be the basis of the smoker's heart. Whatever the mechanism, Auerbach et al have now shown the harmful effect of cigarette smoking on the blood vessels of the heart in specific pathological terms-and their findings should add weight to the medical campaign against tobacco.

${ }^{1}$ British Medical fournal, 1969, 1, 460.

2 Auerbach, O, Hammond, E C, and Garfinkel, L, New England fournal of Medicine, 1965, 273, 775.

${ }^{3}$ Auerbach, O, et al, Archives of Internal Medicine, 1977, 137, 435.

4 Auerbach, O, Hammond, E C, and Garfinkel, L, New England fournal of Medicine, 1968, 278, 980.

5 Auerbach, O, Hammond, E C, and Garfinkel, L, Archives of Environmental Health, 1971, 22, 20.

${ }^{6}$ Hammond, E C, et al, Environmental Research, 1976, 12, 263.

7 Van Vliet, P D, Burchell, H B, and Titus, J L, New England fournal of Medicine, 1966, 274, 1102

\section{Adoption of deprived children}

The number of healthy infants offered for adoption has declined in the past ten years; so adoption agencies have turned their attention to the needs of older children and those with medical or social difficulties. In North America the proportion of older children placed for adoption has greatly increased, and there is a similar trend in Britain. A survey of children in the care of local authorities published in 1974 suggested that about 7000 children, many of them past infancy, should be released for adoption. ${ }^{1}$

Most older children (and some infants too) experience a period of maternal deprivation before going to adopting parents. They have often been looked after by a variety of people and have moved from place to place, and many have been let down or rejected. Their management creates special difficulties, but successful placement is nevertheless more than ever important. It has been said" that "a failed adoption is 